\title{
Efficacy of CR4056, a first-in-class imidazoline-2 analgesic drug, in comparison with naproxen in two rat models of osteoarthritis
}

This article was published in the following Dove Press journal:

Journal of Pain Research

4 May 2017

Number of times this article has been viewed

\author{
Eleonora Comi ${ }^{1,2}$ \\ Marco Lanza' \\ Flora Ferrari' \\ Valeria Mauri ${ }^{1,3}$ \\ Gianfranco Caselli' \\ Lucio Claudio Rovati' \\ 'Department of Pharmacology and \\ Toxicology, Rottapharm Biotech, ${ }^{2} \mathrm{PhD}$ \\ Program in Neuroscience, University \\ of Milan-Bicocca, ${ }^{3}$ Department of \\ Surgery and Translational Medicine, \\ University of Milan-Bicocca, \\ Monza, Italy
}

Purpose: CR4056, (2-phenyl-6-(1H-imidazol-1yl) quinazoline), an imidazoline-2 (I2) receptor ligand, is a promising analgesic drug that has been reported to be effective in several animal models of pain. The aim of this study was to evaluate the effects of CR4056 in two well-established rat models of osteoarthritis (OA), mimicking the painful and structural components of human OA. Methods: Knee OA was induced either by single intra-articular injection of monoiodoacetate (MIA) or by medial meniscal tear (MMT) in the right knee of male rats. In the MIA model, allodynia and hyperalgesia were measured as paw withdrawal threshold to mechanical stimulation. In the MMT model, pain behavior was analyzed as weight-bearing asymmetry (i.e. difference in hind paw weight distribution, HPWD) between the injured and the contralateral limbs. Results: Acute oral administration of CR4056, 14 days after MIA injection, significantly and dose-dependently reduced allodynia and hyperalgesia 90 minutes after treatment, whereas acute naproxen administration significantly reduced allodynia but not hyperalgesia. After 7 days of repeated treatment, both CR4056 and naproxen showed significant anti-allodynic and anti-hyperalgesic effects in the MIA model. Rats undergoing MMT surgery developed a significant and progressive asymmetry in HPWD compared with sham-operated animals. Repeated treatment with CR4056 significantly reduced the progression of the pain behavior, whereas naproxen had no effects.

Conclusion: The data presented here show that the I2 ligand CR4056 could be a new effective treatment for OA pain. The compound is currently under Phase II clinical evaluation for this indication.

Keywords: osteoarthritis, pain, CR4056, imidazoline-2 receptors, MIA, MMT

\section{Introduction}

The main clinical symptoms of osteoarthritis (OA) are joint pain, impaired physical function, and stiffness. ${ }^{1}$ OA pain represents the most disabling symptom for patients and it is the most prevalent form of chronic musculoskeletal pain. In particular, OA pain is defined by peripheral and central sensitization and is driven by both nociceptive and neuropathic mechanisms. ${ }^{2}$ It has long been established that there is poor correlation between the clinical symptoms of OA and joint structure changes, radiologically assessed. ${ }^{3}$ Although important advances have been made in understanding the pathophysiological process of OA, there are currently no approved pharmacological treatments indicated to modify the disease progression. At present, OA pharmacological treatments, along with physical treatments such as physiotherapy and rehabilitation, still focus on improving the disease symptoms and particularly joint pain. ${ }^{4}$ However,
Department of Pharmacology and Toxicology, Rottapharm Biotech, via Valosa di Sopra 9, 20900 Monza, Italy Tel+3939906 6094

Fax +39 399066165

Email marco.lanza@rottapharmbiotech. com 
the efficacy of "first-line" analgesics such as acetaminophen is limited. Moreover, the most common and effective pharmacological therapies, such as nonsteroidal anti-inflammatory drugs (NSAIDs) and stronger analgesics such as opioids, are frequently associated with significant adverse effects. Therefore, more effective approaches to pain management in this condition are needed. ${ }^{5}$

Interestingly, chronic musculoskeletal pain is associated with a dysregulation of the descending inhibitory pathways that modulate pain sensations. ${ }^{6}$ Selective serotonin and norepinephrine reuptake inhibitors such as duloxetine are effective in OA pain, but their use is limited by adverse effects. ${ }^{7}$ Imidazoline-2 (I2) receptors are a potentially more interesting target to affect pain-descending inhibitory pathways in that $\mathrm{I} 2$ receptor ligands have been associated with an allosteric inhibition of monoamine oxidase (MAO), ${ }^{8,9}$ thus increasing the synaptic levels of noradrenaline and serotonin in a more targeted fashion which is different from traditional direct MAO inhibition or reuptake inhibition. Therefore, compounds able to interact with $\mathrm{I} 2$ receptors and thus with MAO activity in an innovative fashion could represent a promising new class of drugs for the treatment of chronic and in particular musculoskeletal pain. ${ }^{10,11}$ Indeed I 2 receptors, also referred to as $\mathrm{I} 2$ binding sites, ${ }^{12}$ are widely distributed in mammalian cells of the central and peripheral nervous system, ${ }^{13}$ and their ligands have been suggested as putative analgesics, especially in tonic inflammatory and neuropathic pain models. ${ }^{14}$

CR4056 (2-phenyl-6-(1H-imidazol-1yl) quinazoline; Rottapharm Biotech, Monza, Italy) is a novel 12 receptor ligand that binds to $\mathrm{I} 2$ sites with a sub-micromolar affinity, and via this interaction it inhibits human recombinant as well as rat MAO-A activity through an allosteric mechanism. ${ }^{15}$

Notably, orally administered CR4056 almost invariably reversed the hyperalgesia observed in several animal models of pain including chronic (complete Freund's adjuvant) or neurogenic (capsaicin) inflammation, ${ }^{15}$ postoperative pain, ${ }^{16}$ neuropathies (diabetes, chemotherapy), ${ }^{15,17}$ and non-inflammatory muscular pain mimicking the symptoms of human fibromyalgia. ${ }^{15}$ Preclinical data on CR4056 in animal models of OA pain were not available before this study. The in vivo pharmacology underlying the analgesic activity of CR4056 was examined in models of pain induced by capsaicin ${ }^{15}$ or surgery, ${ }^{16}$ where the $\mathrm{I} 2$ antagonist idazoxan - but not $\alpha 1, \alpha 2$, or I1 receptor antagonists - suppressed the effect of a fully active dose of CR4056. This finding is important, because it strongly suggests that interaction with I 2 sites is the primary requirement for the analgesic activity of CR4056 to take place. CR4056 has no affinity for opioid receptors, but it evidenced a relevant synergy with morphine in both the pain models we used to study the in vivo pharmacology of this compound. ${ }^{15,16}$ In the present study, we focused on CR4056 ability to treat OA pain in relevant animal models of the disease. This is the set of experiments that allowed moving the compound into Phase II clinical trials for knee OA pain. We selected both the monosodium iodoacetate (MIA) model and the medial meniscal tear (MMT) model in rats as two commonly used OA animal models able to mimic the painful and structural components of the human pathology.

Using these two models of OA-like pain behavior, we investigated the characteristics of oral CR4056 either as an acutely acting analgesic, after single administration, or as a long-acting symptom modifier, after sub-acute daily administrations ( 7 or 14 days in MIA and MMT, respectively). The analgesic properties of CR4056 were compared with those of naproxen, an NSAID analgesic commonly prescribed for human OA pain.

\section{Materials and methods}

\section{Animals, drugs, and treatments}

Male Wistar rats (Harlan, S. Pietro al Natisone, Italy) weighing 150-175 g and male Sprague Dawley rats (Charles River Laboratories, Calco, Italy) weighing 300-325 g were housed with ad libitum access to food and water, in a temperaturecontrolled room with a 12 hours light/dark cycle, at least 1 week before the beginning of the experimental setup. In order to set up the experimental conditions, we performed a series of preliminary experiments in which a total of 96 animals were used (see Figures S1 and S2, Tables S1 and S2 for details and results). However, in the experiments described here, a total of 66 animals were used. All the experimental procedures described were in compliance with international laws and policies (Directive 2010/63/EU on the protection of animals used for scientific purposes; Guide for the Care and Use of Laboratory Animals, U.S. National Research Council, 1996) and were approved by the Italian Ministry of Health. All studies involving animals are reported in accordance with the ARRIVE guidelines for reporting experiments involving animals. ${ }^{18,19}$

CR4056 was suspended in $0.5 \%$ hydroxyl-propylcarboxymethyl cellulose (HPMC) and administered orally in $5 \mathrm{~mL} / \mathrm{kg}$ administration volume. Naproxen sodium salt (Sigma Aldrich, Milan, Italy) was dissolved in distilled water and administered orally in $5 \mathrm{~mL} / \mathrm{kg}$ administration volume.

As regards the MIA model, CR4056 (2 and $6 \mathrm{mg} / \mathrm{kg}$ ) and naproxen $(10 \mathrm{mg} / \mathrm{kg})$ were administered from day 14 to 
day 21, once daily. Similarly, MMT rats were treated orally with CR4056 (6 mg/kg) and naproxen $(10 \mathrm{mg} / \mathrm{kg})$ from day 28 to day 42, once daily. Sham and control animals were orally administered with HPMC (vehicle) following the same schedule.

\section{MIA model of OA pain}

Unilateral knee OA was induced by a single intra-articular injection of MIA (Sigma Aldrich) in the infrapatellar area of the right knee of male Wistar rats. A total of 30 animals were used (six rats per group). The experimental procedure was performed according to the method first proposed by Kalbhen, ${ }^{20}$ and later revised by Sagar et al. ${ }^{21}$ The dose of MIA was selected based on a combination of preliminary results and literature data. In particular, we noticed that $1 \mathrm{mg}$ MIA was the minimum dose required to detect secondary allodynia and primary hyperalgesia associated with joint damage. Therefore, this dose was selected for the present study. On day 1 , rats were anesthetized with $2 \%$ isoflurane in pure oxygen. The right leg skin was sterilized with $75 \%$ ethyl alcohol and the knee was located by palpation. A 26-gauge needle was inserted vertically to penetrate the skin, and turned distally for insertion into the articular cavity, until a distinct loss of resistance was detected; $1 \mathrm{mg}$ of MIA in $50 \mu \mathrm{L}$ saline was then delivered into the articular cavity. Control rats (sham) received $50 \mu \mathrm{L}$ of saline solution. All animals were sacrificed 21 days after the intra-articular injection of either MIA or saline solution.

\section{MMT model of OA}

Unilateral knee OA was induced by transection of both the medial collateral ligament and the medial meniscus of the right knee of male Sprague Dawley rats, which results in a progressive cartilage degeneration and several joint changes typical of OA. ${ }^{22} \mathrm{~A}$ total of 36 animals were used (nine rats per group). On day 1 , rats were anesthetized with $2 \%$ isoflurane in pure oxygen. The right leg was shaved and the skin was sterilized with $75 \%$ ethyl alcohol. The medial collateral ligament was exposed by blunt dissection and transected to reflect the meniscus toward the femur. The joint space was visualized and the meniscus was cut through the full thickness at its narrowest point to simulate a complete tear, taking care not to damage the tibial surface. The connective tissue layer and skin were closed with Vicryl 6.0 suture (Ethicon, Sommerville, NJ, USA) and a skin glue (3M Vetbond Tissue Adhesive; $3 \mathrm{M}$ Science. Applied to Life, St. Paul, MN, USA), respectively. Sham-operated animals underwent only a skin incision. After surgery, rats were housed three per cage Eurostandard type-IV cages (Tecniplast SpA, Buguggiate,
VA, Italy), measuring $595 \times 380 \times 200 \mathrm{~mm}$. All animals were sacrificed 6 weeks after MMT surgery, an experimental time period sufficient to create severe joint lesions that simulate late-stage $\mathrm{OA}$ as demonstrated by several previous studies. ${ }^{22,23}$

\section{Behavioral assessments}

Behavioral assessments were selected according to previous reports related to pain evaluation in animal models of OA. ${ }^{24,25}$ Scientists performing behavioral experiments were always blind to treatments. Two behavioral tests were used to determine the alterations in pain perception after MIA injection. Namely, we compared the effect of CR4056 versus naproxen by evaluating both the allodynic and the hyperalgesic responses to mechanical stimuli by the von Frey and the pressure application measurement (PAM) tests, respectively (see the following texts). The behavioral assessments were performed on day 1 before MIA injection and on days 7, 14, and 21 after MIA injection. On days 14 and 21, behavioral tests were performed 90 minutes after the drug administration because in-house preliminary experiments selected this time as the optimal time to observe reversal of pain behavior after the oral drug administration. As regards MMT surgery, joint discomfort perception was evaluated as an indirect index of spontaneous pain by assessing changes in hind paw weightbearing distribution. The behavioral measure was performed 1 day before MMT surgery; on days 14, 28, 35, and 42 after the induction of OA; and before and 90 minutes after the drug administration.

\section{Von Frey test}

Mechanical allodynia was assessed using a Dynamic Plantar Aesthesiometer (Ugo Basile S.r.1., Gemonio, VA, Italy), which generated a linearly increasing mechanical force. ${ }^{26}$ On each day of testing, rats were placed in a Plexiglas chamber $(28 \times 40 \times 35 \mathrm{~cm})$ equipped with a wire mesh floor, $20 \mathrm{~cm}$ above the bench, for an acclimatization period of 1 hour, followed by testing. After the habituation period, a servo-controlled mechanical stimulus, represented by a pointed metallic filament of $0.5 \mathrm{~mm}$ diameter, was positioned under the plantar surface of the hind paw, exerting a progressively increasing punctuate pressure, reaching up to $50 \mathrm{~g}$ within 20 seconds. A clear spontaneous hind paw withdrawal response caused by the punctuate pressure was recorded automatically. The results defined the minimum pressure (in grams) required to elicit a robust and immediate withdrawal reflex of the paw. Stimuli were applied alternatively on each hind paw every 2 minutes on three repeated measures to obtain a mean value representing the mechanical allodynic threshold. 


\section{PAM test}

Mechanical hyperalgesia was assessed using the PAM device (Ugo Basile S.r.1.), a force transducer (range: 0-1500 $\mathrm{g}$ and $8 \mathrm{~mm}$ diameter) mounted on a unit fitted to the operator's thumb and connected to a recording base unit. ${ }^{27}$ Animals were lightly, but securely, held and the operator placed the thumb unit on one side of the animal knee joint and the forefinger on the other. A progressive quantified squeeze force was applied across the joint at a rate of approximately 300 grams per second ( 5 seconds as maximum test duration). The force that elicits the animal response, normally knee withdrawal or any behavioral signs of discomfort or distress, such as freezing of whisker movement or wriggling, was recorded. In particular, the peak gram force applied immediately prior to knee withdrawal, designated as limb withdrawal threshold, represents the mechanical hyperalgesic threshold.

\section{Incapacitance test}

Changes in hind paw weight distribution (HPWD) between the right (ipsilateral control) and left (contralateral control) limbs were utilized as an index of joint discomfort in the osteoarthritic knee. ${ }^{28}$ HPWD was assessed using an incapacitance tester (Linton Instrumentation, Norfolk, UK). The apparatus consists of two force transducers capable of measuring the body weight that the animal places on each hind knee. Animals were placed in an angled plexiglass chamber positioned so that each hind paw rested on a separate force plate; the force exerted by each hind knee (in grams) was calculated over a 5-second period. For each animal two 5 -second readings were taken. The change in HPWD was calculated by determining the difference in the amount of weight ( $\mathrm{g}$ ) between the left (healthy-contralateral) and right (operated-ipsilateral) paw. The efficacy of pharmacological treatments was evaluated as percent difference between $\Delta$ weight measured at the beginning of treatments (i.e. day 28 after MMT surgery) and at the end of 2-weeks of treatment (i.e. day 42 after MMT surgery).

\section{Statistical analysis}

Data analysis was performed on the absolute mechanical threshold values (MIA model; crude values) or on the percent difference in weight bearing between the contralateral and ipsilateral knees (MMT model; normalized values in order to control for unwanted sources of variation). Data were analyzed by two-way mixed model ANOVA, with $P<0.05$ accepted as significant (SigmaStat 3.1; Systat Software Inc., San Jose, CA, USA). Multiple inter-group differences were assessed by the Holm-Sidak method. Data are presented as mean \pm standard error. Statistical significance was calculated by one-way ANOVA followed by Tukey's multiple comparisons test.

\section{Results MIA model of OA pain}

Intra-articular injection of $1 \mathrm{mg} / 50 \mu \mathrm{L}$ MIA induced significant mechanical allodynia (von Frey test) of the ipsilateral paw compared with saline controls or with the contralateral paw (Figure 1). Rats injected with MIA maintained this significant decrease in withdrawal threshold in the ipsilateral paw up to 21 days after MIA injection. No variations in withdrawal threshold of the contralateral paw were observed in both saline- and MIA-treated rats, throughout the course of the study. Similar results were obtained with the assessment of mechanical hyperalgesia at the PAM test (Figure 2). MIA injection caused a long-lasting decrease of withdrawal threshold in the ipsilateral limb compared with saline controls or with the contralateral limb. Knee withdrawal thresholds detected in the contralateral knee showed no variations in both sham and control rats injected with MIA, throughout the study.

CR4056 (2 and $6 \mathrm{mg} / \mathrm{kg}$ ) and naproxen (10 mg/kg) were administered orally starting on day 14 after MIA injection. Paw and knee withdrawal thresholds were evaluated 90 minutes posttreatment in both experimental settings. The assessment of mechanical allodynia showed an increase in the ipsilateral paw withdrawal threshold after acute oral treatment (day 14) with both $6 \mathrm{mg} / \mathrm{kg} \mathrm{CR} 4056$ and $10 \mathrm{mg} /$ $\mathrm{kg}$ naproxen compared with vehicle (Figure 1). CR4056 (6 $\mathrm{mg} / \mathrm{kg}$ ) induced also a significant reversal of MIA-induced hyperalgesia (day 14) (Figure 2), whereas naproxen and the lower dose of CR4056 showed a trend of efficacy not reaching statistical significance. The pharmacological treatments did not influence the withdrawal threshold of the contralateral paw or knee (data not shown).

The efficacy of $6 \mathrm{mg} / \mathrm{kg}$ CR4056 was further confirmed on day 21 after the animals received daily treatments from day 14 to day 21, both on allodynia and hyperalgesia (Figures 1 and 2). On day 21 the statistical significance was reached also by the lower dose of CR4056 on hyperalgesia (Figure 2). Naproxen was effective on both parameters too.

\section{MMT model of OA}

Transection of the medial collateral ligament and medial meniscus of the knee induced a significant and time-dependent development of weight-bearing asymmetry compared with sham-operated animals (Figure 3A). Indeed, MMT 


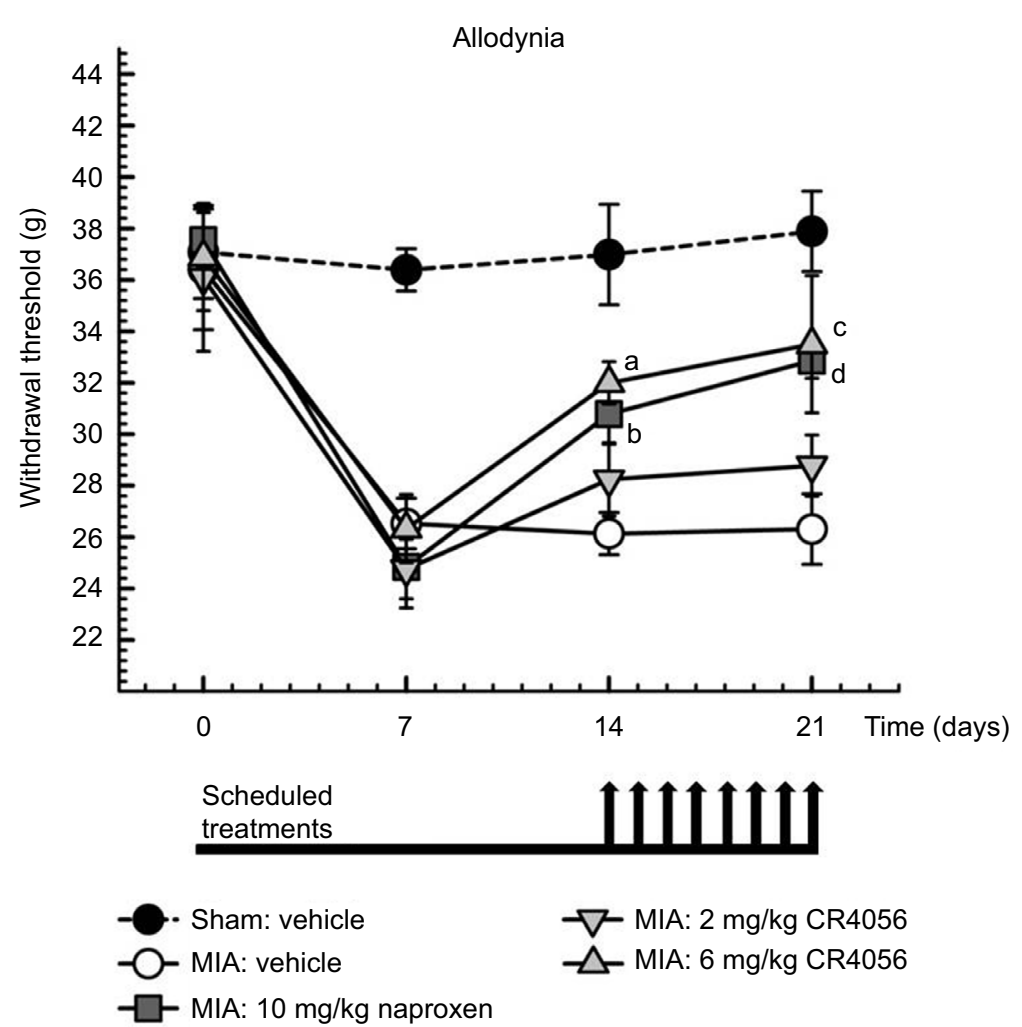

Figure I MIA model of OA pain: effect of CR4056 and naproxen on mechanical allodynia. Rats were injected with I mg/50 $\mu$ l of MIA or saline (sham group) in the right knee. Mechanical withdrawal threshold (in grams) was evaluated in the ipsilateral paw by von Frey test, using a Dynamic Plantar Aesthesiometer, prior and 7, I4, and 21 days after MIA injection. CR4056 (2 and $6 \mathrm{mg} / \mathrm{kg})$ and naproxen $(10 \mathrm{mg} / \mathrm{kg})$ were administered orally, once a day under the schedule indicated by the arrows positioned in the graph below the axis of time (days). Data, collected 90 minutes after the drug administration, represent the mean \pm SEM of six animals per group.

Notes: ${ }^{\mathrm{a}} P=0.007,{ }^{\mathrm{b}} \mathrm{P}=0.03 \mathrm{I},{ }^{\mathrm{c}} \mathrm{P}=0.00 \mathrm{I},{ }^{\mathrm{d} P} \mathrm{P}=0.003$ versus MIA group treated with vehicle; two-way mixed model ANOVA, Holm-Sidak test.

Abbreviations: MIA, monoiodoacetate; OA, osteoarthritis; ANOVA, one-way analysis of variance, SEM, standard error of the mean.

surgery increased joint discomfort as defined by a significant and progressive increase in the difference in HPWD between the ipsilateral and contralateral knees throughout the course of the study.

CR4056 $(6 \mathrm{mg} / \mathrm{kg})$ and naproxen $(10 \mathrm{mg} / \mathrm{kg})$ were administered orally starting on day 28 after MMT surgery. HPWD was measured before and 90 minutes posttreatment. The acute administration of CR4056 and naproxen (day 28) did not change the left-right hind paw weight-bearing asymmetry compared with vehicle-treated rats (Figure 3A).

CR4056 and naproxen were further administered orally as sub-acute treatments from day 28 to day 42 after MMT surgery, once daily. The assessment of HPWD 7 days after the beginning of treatments (day 35) showed a significant reduction in the right-left weight-bearing asymmetry in rats treated with $6 \mathrm{mg} / \mathrm{kg}$ CR4056 (Figure 3A). Conversely, MMT rats treated for 7 days (day 35) with $10 \mathrm{mg} / \mathrm{kg}$ naproxen were not different from rats treated with vehicle. After 14 days of treatment (day 42) HPWD results confirmed those obtained 7 days before, that is CR4056 treatment significantly blocked the pain progression whereas naproxen showed only a non-significant tendency toward the reduction of the weight-bearing asymmetry. Notably, the asymmetry of animals treated with CR4056 on day 42 after surgery was significantly lower than that of the same animals before starting the treatment (day 28 after surgery) (Figure 3B), thus suggesting that CR4056 is not only able to counteract the progression of the symptom seen between days 28 and 42 after surgery but also able to reduce it.

\section{Discussion}

In the present study, the $\mathrm{I} 2$ receptor ligand CR 4056 potently and dose-dependently relieved pain in two experimental models of knee OA, after both acute and especially chronic administrations and with a pattern that tended to be superior to the NSAID naproxen.

Efficacy evaluation of an analgesic treatment for a chronic condition such as OA deserves caution due to objective translational challenges when considering animal models in which OA has been induced acutely. ${ }^{29}$ This is the reason why we chose two well-known and quite different animal models of OA in the same species: the chemically induced MIA model and surgically induced meniscal transection (MMT) in rats. The two models have been extensively characterized 


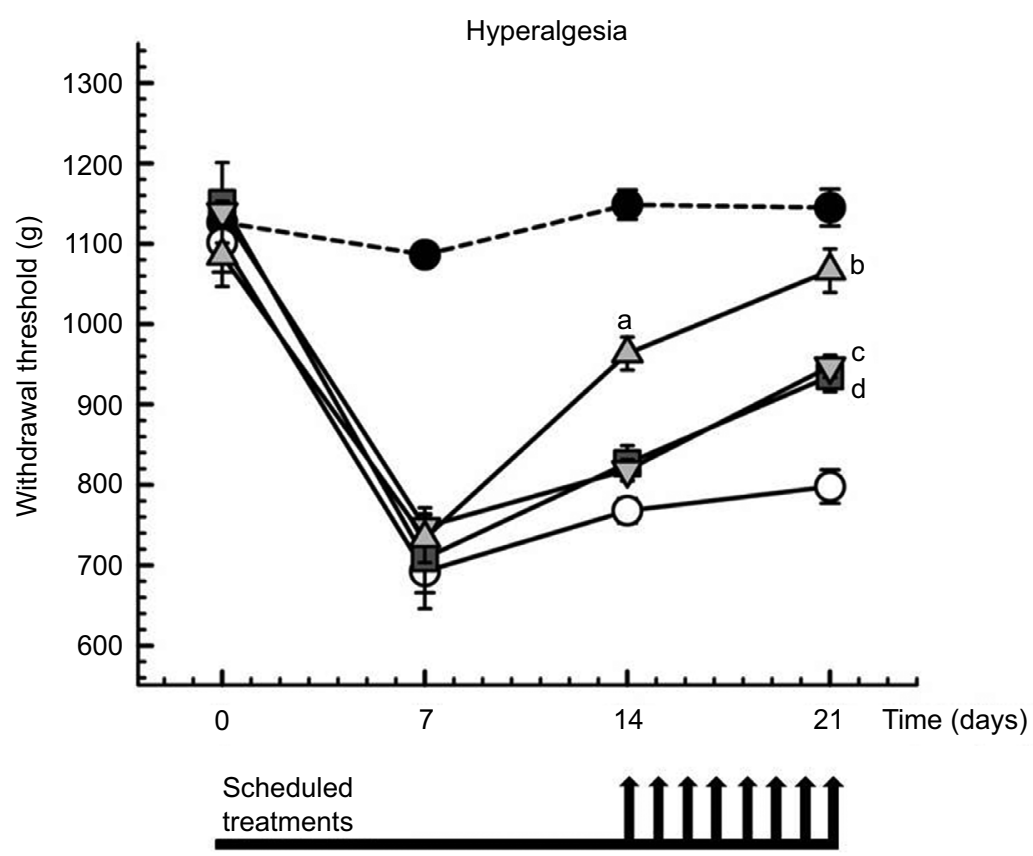

- Sham: vehicle
- MIA: vehicle
$\square$ MIA: $10 \mathrm{mg} / \mathrm{kg}$ naproxen

Figure 2 MIA model of OA pain: effect of CR4056 and naproxen on mechanical hyperalgesia. Rats were injected with I mg/50 $\mu$ l of MIA or saline (sham group) in the right knee. Mechanical withdrawal threshold (in grams) was evaluated in the ipsilateral knee using a Pressure Application Measurement (PAM) device, prior and 7, I4, and 21 days after MIA injection. CR4056 (2 and $6 \mathrm{mg} / \mathrm{kg})$ and naproxen $(10 \mathrm{mg} / \mathrm{kg})$ were administered orally, once a day under the schedule indicated by the arrows positioned in the graph below the axis of time (days). Data, collected 90 minutes after the drug administration, represent the mean \pm SEM of six animals per group.

Notes: ${ }^{a} P<0.001,{ }^{b} P<0.001,{ }^{c} P<0.001,{ }^{d} P=0.001$ versus MIA group treated with vehicle; two-way mixed model ANOVA, Holm-Sidak test.

Abbreviations: MIA, monoiodoacetate; OA, osteoarthritis; ANOVA, one-way analysis of variance, SEM, standard error of the mean.

A

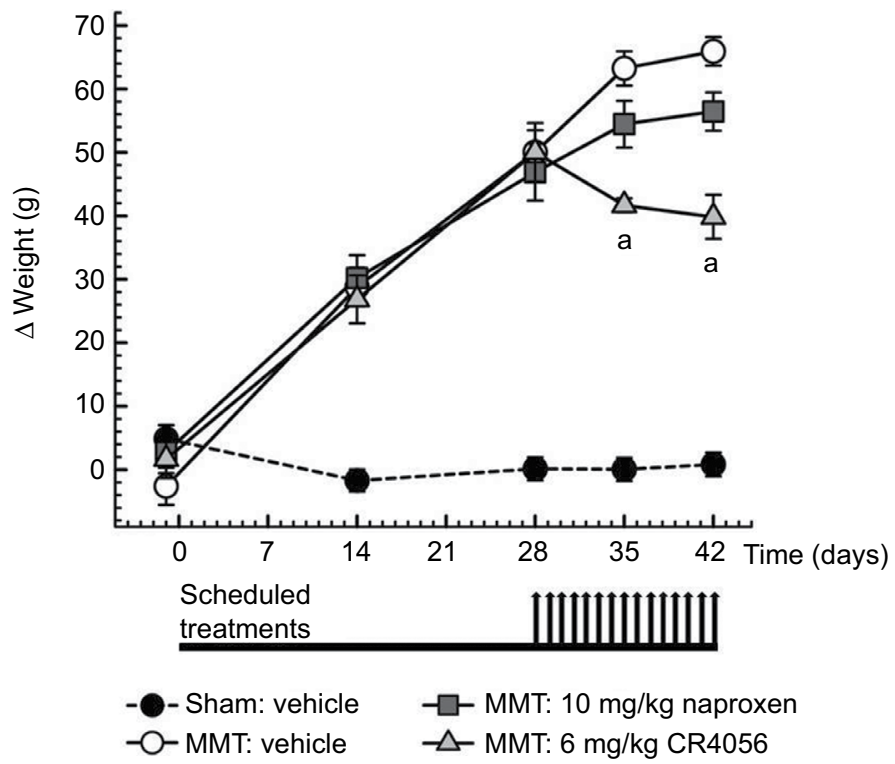

B

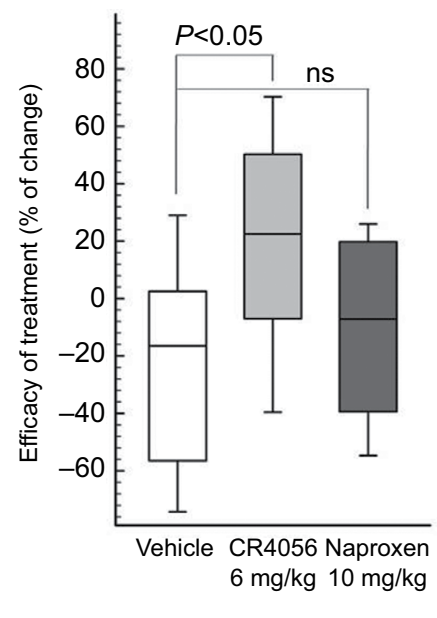

Figure 3 MMT model of OA: effect of CR4056 and naproxen on the behavioral assessment of hind paw weight distribution (HPWD). (A) The difference in HPWD ( $\triangle$ ) between contralateral and ipsilateral knees (in grams) was evaluated I day before and 14, 28, 35, and 42 days after MMT surgery using an incapacitance tester. CR4056 $(6 \mathrm{mg} / \mathrm{kg})$ and naproxen $(10 \mathrm{mg} / \mathrm{kg}$ ) were administered orally, once a day under the schedule indicated by the arrows positioned in the graph below the axis of time (days). Data, collected 24 hours after the drug administration, represent the mean \pm SEM of nine animals per group. ${ }^{a}<<0.00$ I versus MMT control rats treated with vehicle; two-way mixed model ANOVA, Holm-Sidak test. (B) Efficacy of 2 weeks of treatment with CR4056 $(6 \mathrm{mg} / \mathrm{kg})$ and naproxen $(10 \mathrm{mg} / \mathrm{kg})$ assessed as percent difference between $\Delta$ weight measured at day 28 (before the beginning of treatments) and day 42 after MMT surgery. Data ( $\mathrm{N}=9$ animals/group) were plotted as box-and-whisker plot. The box spans the interquartile range (25th-75th percentiles), while the line within the box denotes the median. Whiskers extend from the I0th to the 90th percentiles. Statistical significance was calculated by one-way ANOVA followed by Tukey's multiple comparisons test.

Abbreviations: MMT, medial meniscal tear; OA, osteoarthritis; ANOVA, one-way analysis of variance, SEM, standard error of the mean; ns, not significant. 
and compared, ${ }^{25}$ showing that weight-bearing asymmetry increased over time in the surgical model whereas this did not occur in the MIA model. Conversely, withdrawal thresholds were reduced in MIA rats but not in MMT rats. The authors discussed these results suggesting that the relative contribution of peripheral and central pain mechanisms may differ significantly between these two models. ${ }^{25}$ Other groups reported both inflammation of the knee and central sensitization when the dose of MIA is increased, ${ }^{30}$ as well as significant differences when evaluating the allodynic response in MMT rats compared to sham-operated controls. ${ }^{24,28}$ Notably, the HPWD paradigm in MIA appears so variable that the same authors report its insensitivity or its sensitivity in two different papers. ${ }^{25,31}$ In fact, our preliminary experiments, while confirming the relative insensitivity of MMT evaluation by von Frey (Figure S1 and Table S1) that renders this paradigm unsuitable for testing analgesic compounds, gave a significant, albeit if very variable HPWD response in the MIA paradigm, and a significant effect of CR4056 also in this preliminary experiment (Figure S2 and Table S2). Therefore, to evaluate the efficacy of a novel analgesic in OA models, we chose to limit behavioral assessments to those more widely accepted by groups working on OA pain, that is mechanical paw withdrawal threshold in MIA and HPWD analysis in MMT rats, respectively. This apparent limitation of the study had the advantage of decreasing the total number of behavioral trials carried out in the course of the study, thus contributing to reduce the stress of the animals and, consequently, to improve their ability to adequately respond to the stimulus. ${ }^{32}$

Consistently with its analgesic efficacy previously evidenced in different animal models, ${ }^{11,15-17,33}$ CR4056 showed a significant and dose-dependent anti-hyperalgesic and antiallodynic effect both following acute and repeated daily treatment from day 14 to day 21 after MIA injection. Oral CR4056 reduced also hind limb weight-bearing imbalance after repeated daily treatment from day 28 to day 42 after MMT surgery. Consistently with what happens in the human disease, ${ }^{2,34}$ both an inflammatory component and a neuropathic component of pain appear to be involved in these experimental paradigms, ${ }^{35}$ where joint damage is induced by transection of the medial collateral ligament and medial meniscus of the femoro-tibial joint and by intra-articular injection of MIA, respectively, with consequent pain behavior.

Naproxen was used as a positive comparator. Acute naproxen was previously found to alleviate the joint discomfort following MIA injection in the rat. ${ }^{28}$ Other authors suggest a specific role for NSAIDs only during the first week post-MIA injection. ${ }^{36,29} \mathrm{In}$ our hands, $10 \mathrm{mg} / \mathrm{kg}$ naproxen evidenced a robust efficacy to reverse allodynia both after acute administration and after 7 days of repeated treatment, comparable to that of $6 \mathrm{mg} / \mathrm{kg}$ CR4056. Conversely, when testing naproxen for anti-hyperalgesic efficacy after acute administration, we found only a trend of efficacy that did not reach statistical significance, contrary to CR4056. However, after 7 days of repeated treatment, naproxen evidenced a significant anti-hyperalgesic effect, although at a lower level than CR4056. On the other hand, and again differently than CR4056, naproxen was not effective when tested in rats undergoing MMT surgery after 7 or 14 days of repeated treatment. To the best of our knowledge, there are no studies in the literature using naproxen as NSAID analgesic medication in the rat MMT model of OA, despite naproxen being one of the preferred NSAIDs for long-term conditions such as OA, and very few studies even using different NSAIDs. ${ }^{23,37}$

We hypothesize that the potent analgesic effects of CR4056 are primarily mediated by its interaction with I2 binding sites, since the effects in capsaicin- or surgeryinduced pain models were suppressed by the putative I2R antagonist idazoxan, but not by $\alpha 1, \alpha 2$, or I1 receptor antagonists. ${ }^{15,16}$ To our knowledge, this is the first evidence that an I 2 ligand could effectively counteract pain behaviors induced by OA experimental models.

Another relevant activity mediated by $\mathrm{I} 2$ receptors is the modulation of the opioid system, which may occur at different levels. The interaction between I 2 and opioid receptors is probably involved in the prevention of tolerance and addiction to opioids by $\mathrm{I} 2$ receptor ligands, ${ }^{38}$ and may further support their efficacy e.g. in chronic pain when used as monotherapy, or combined with opioids as an adjuvant, also decreasing their side effects. ${ }^{17}$ However, the opiate antagonist naloxone was not able to block the analgesic effects of CR4056, ${ }^{15}$ nor CR4056 has any affinity toward opiate receptors. Only the hypoalgesic component (not observed in the present study) of the overall analgesic activity of CR4056 may be indirectly mediated through I2A-dependent idazoxan-insensitive release of endogenous endorphins, ${ }^{15,16,39}$ and such cross-talk should be further investigated.

\section{Conclusion}

In summary, we found that CR4056 significantly reduced the progression of pain-like behaviors in two different rat models of OA, without apparent tolerance after repeated doses. Notably, because the most relevant pain-like behavioral data on MMT rats were obtained 24 hours after the last treatment, it seems reasonable to speculate about a true modification of pain perception. Overall, while in vehicle- and in naproxentreated animals, pain continued to progress, CR4056 was able to significantly reduce it. 
Thus, if the predictive value of the experimental models used herein is confirmed, CR4056 could represent a new highly effective treatment option for OA pain. The compound is currently under Phase II clinical evaluation for this indication.

\section{Acknowledgments}

The authors wish to thank Dr. Albino Bonazzi for his institutional reporting and useful discussion on this project, Dr. Paolo Garofalo for his help in statistical analysis, Mr. Luca Catapano and Mr. Dario Tremolada for their skilful technical help in settling the experimental conditions, and Mrs. Laura Radaelli for secretarial assistance. Preliminary results of this study were presented at the 2015 World Congress on Osteoarthritis (OARSI), held in Seattle, WA, USA (Caselli et al, Osteoarthritis Cartilage, 2015), at the Fifth International Congress on Neuropathic Pain (NeuPSIG) held in Nice, France (Comi et al, 2015), and at the 2016 World Congress on Osteoarthritis (OARSI), held in Amsterdam, the Netherlands (Comi et al, Osteoarthritis Cartilage, 2016). This study was funded by Rottapharm Biotech.

\section{Author contributions}

All authors contributed toward data analysis, drafting and revising the paper and agree to be accountable for all aspects of the work.

\section{Disclosure}

This manuscript has not been published and is not under consideration for publication elsewhere. Rottapharm Biotech as a corporate entity had no role in the conduct of the study, in the collection, analysis, and interpretation of data, in the writing of the report, or in the decision to submit the paper for publication.

Eleonora Comi is a $\mathrm{PhD}$ student with no competing interests. Valeria Mauri is a postgraduate student with no competing interests. All the other authors are employees of Rottapharm Biotech and report no other conflicts of interest in this work.

\section{References}

1. Buchanan WW, Kean WF. Osteoarthritis II: pathology and pathogenesis. Inflammopharmacology. 2002;10:23-52.

2. Salaffi F, Ciapetti A, Carotti M. The sources of pain in osteoarthritis: a pathophysiological review. Reumatismo. 2014;66(1):57-71.

3. Kean WF, Kean R, Buchanan WW. Osteoarthritis: symptoms, signs, and source of pain. Inflammopharmacology. 2004;12(1):3-31.

4. Sofat N, Kuttapitiya A. Future directions for the management of pain in osteoarthritis. Int J Clin Rheumatol. 2014;9(2):197-276.

5. Malfait AM, Schnitzer TJ. Towards a mechanism-based approach to pain management in osteoarthritis. Nat Rev Rheumatol. 2013;9(11):654-664.
6. Fitzcharles MA, Shir Y. Management of chronic pain in the rheumatic diseases with insights for the clinician. Ther Adv Musculoskelet Dis. 2011;3(4):179-190.

7. McAlindon TE, Bannuru RR, Sullivan MC, et al. OARSI guidelines for the non-surgical management of knee osteoarthritis. Osteoarthritis Cartilage. 2014;22(3):363-388.

8. Tesson F, Limon-Boulez I, Urban P, et al. Localization of I2-imidazoline binding sites on monoamine oxidases. J Biol Chem. 1995; 270(17):9856-9861.

9. Ozaita A, Olmos G, Boronat MA, Lizcano JM, Unzeta M, GarcíaSevilla JA. Inhibition of monoamine oxidase A and B activities by imidazol(ine)/guanidine drugs, nature of the interaction and distinction from I2-imidazoline receptors in rat liver. Br J Pharmacol. 1997; 121(5):901-912.

10. Kuner R. Central mechanisms of pathological pain. Nat Med. 2010; 16(11):1258-1266.

11. Li JX, Zhang Y. Emerging drug targets for pain treatment. Eur J Pharmacol. 2012;681(1-3):1-5.

12. Alexander SP, Davenport AP, Kelly E, et al. The Concise Guide to PHARMACOLOGY 2015/16: G protein-coupled receptors. Br $J$ Pharmacol. 2015;172(24):5744-5869.

13. Molderings GJ. Imidazoline receptors: basic knowledge, recent advances and future prospects for therapy and diagnosis. Drug Future. 1997;22(7):757-772.

14. Li JX, Zhang Y. Imidazoline I2 receptors: target for new analgesics? Eur J Pharmacol. 2011;658(2-3):49-56.

15. Ferrari F, Fiorentino S, Mennuni L, et al. Analgesic efficacy of CR4056, a novel imidazoline-2 receptor ligand, in rat models of inflammatory and neuropathic pain. J Pain Res. 2011;4:111-125.

16. Lanza M, Ferrari F, Menghetti I, Tremolada D, Caselli G. Modulation of imidazoline I2 binding sites by CR4056 relieves postoperative hyperalgesia in male and female rats. Br J Pharmacol. 2014;171(15): 3693-3701.

17. Meregalli C, Ceresa C, Canta A, et al. CR4056, a new analgesic I2 ligand, is highly effective against bortezomib-induced painful neuropathy in rats. J Pain Res. 2012;5:151-167.

18. Kilkenny C, Browne W, Cuthill IC, Emerson M, Altman DG; NC3Rs Reporting Guidelines Working Group. Animal research: reporting in vivo experiments: the ARRIVE guidelines. Br J Pharmacol. 2010; 160(7):1577-1579.

19. McGrath JC, Drummond GB, McLachlan EM, Kilkenny C, Wainwright CL. Guidelines for reporting experiments involving animals: the ARRIVE guidelines. Br J Pharmacol. 2010;160(7):1573-1576.

20. Kalbhen DA. Chemical model of osteoarthritis - a pharmacological evaluation. J Rheumatol. 1987;14 Spec No:130-131.

21. Sagar DR, Burston JJ, Hathway GJ, et al. The contribution of spinal glial cells to chronic pain behaviour in the monosodium iodoacetate model of osteoarthritis pain. Mol Pain. 2011;7:88.

22. Janusz MJ, Bendele AM, Brown KK, Taiwo YO, Hsieh L, Heitmeyer SA. Induction of osteoarthritis in the rat by surgical tear of the meniscus: inhibition of joint damage by a matrix metalloproteinase inhibitor. Osteoarthritis Cartilage. 2002;10(10):785-791.

23. Bove SE, Laemont KD, Brooker RM, et al. Surgically induced osteoarthritis in the rat results in the development of both osteoarthritislike joint pain and secondary hyperalgesia. Osteoarthritis Cartilage. 2006;14(10):1041-1048.

24. Fernihough J, Gentry C, Malcangio M, et al. Pain related behaviour in two models of osteoarthritis in the rat knee. Pain. 2004;112(1-2):83-93.

25. Mapp PI, Sagar DR, Ashraf S, et al. Differences in structural and pain phenotypes in the sodium monoiodoacetate and meniscal transection models of osteoarthritis. Osteoarthritis Cartilage. 2013;21(9):1336-1345.

26. Malfait AM, Little CB, McDougall JJ. A commentary on modelling osteoarthritis pain in small animals. Osteoarthritis Cartilage. 2013;21(9): 1316-1326.

27. Barton NJ, Strickland IT, Bond SM, et al. Pressure application measurement (PAM): a novel behavioural technique for measuring hypersensitivity in a rat model of joint pain. J Neurosci Methods. 2007;163(1):67-75. 
28. Bove SE, Calcaterra SL, Brooker RM, et al. Weight bearing as a measure of disease progression and efficacy of anti-inflammatory compounds in a model of monosodium iodoacetate-induced osteoarthritis. Osteoarthritis Cartilage. 2003;11(11):821-830.

29. Rashid MH, Theberge Y, Elmes SJ, Perkins MN, McIntosh F. Pharmacological validation of early and late phase of rat mono-iodoacetate model using the Tekscan system. Eur J Pain. 2013;17(2):210-222.

30. Malfait AM, Little CB. On the predictive utility of animal models of osteoarthritis. Arthritis Res Ther. 2015;17:225.

31. Nwosu LN, Mapp PI, Chapman V, Walsh DA. Blocking the tropomyosin receptor kinase $\mathrm{A}$ (TrkA) receptor inhibits pain behaviour in two rat models of osteoarthritis. Ann Rheum Dis. 2016;75(6):1246-1254.

32. Cornélio AM, Mendes-Gomes J, Fugimoto JS, Morgan MM, Nunes-deSouza RL. Environmentally induced antinociception and hyperalgesia in rats and mice. Brain Res. 2011;1415:56-62.

33. Li JX, Thorn DA, Qiu Y, Peng BW, Zhang Y. Antihyperalgesic effects of imidazoline I(2) receptor ligands in rat models of inflammatory and neuropathic pain. Br J Pharmacol. 2014;171(6):1580-1590.
34. Thakur M, Dickenson AH, Baron R. Osteoarthritis pain: nociceptive or neuropathic? Nat Rev Rheumatol. 2014;10(6):374-380.

35. Im HJ, Kim JS, Li X, et al. Alteration of sensory neurons and spinal response to an experimental osteoarthritis pain model. Arthritis Rheum. 2010;62(10):2995-3005.

36. Gregersen LS, Røsland T, Arendt-Nielsen L, Whiteside G, Hummel M. Unrestricted weight bearing as a method for assessment of nociceptive behavior in a model of tibiofemoral osteoarthritis in rats. J Behav Brain Sci. 2013;3:306-314.

37. Ashraf S, Mapp PI, Walsh DA. Contributions of angiogenesis to inflammation, joint damage, and pain in a rat model of osteoarthritis. Arthritis Rheum. 2011;63(9):2700-2710.

38. Thorn DA, Zhang Y, Li JX. Effects of the imidazoline I 2 receptor agonist 2-BFI on the development of tolerance to and behavioural/physical dependence on morphine in rats. Br J Pharmacol. 2016;173(8):1363-1372.

39. Chang CH, Wu HT, Cheng KC, Lin HJ, Cheng JT. Increase of beta-endorphin secretion by agmatine is induced by activation of imidazoline I(2A) receptors in adrenal gland of rats. Neurosci Lett. 2010;468(3):297-299. 


\section{Supplementary materials}

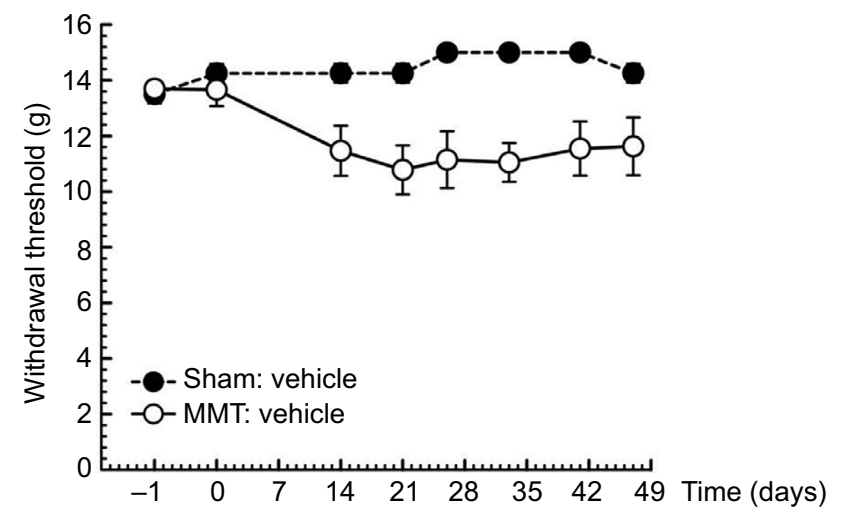

Figure SI Determination of allodynia in the MMT model of OA via manual von Frey analysis. Allodynia was measured via von Frey filaments in SD rats in which OA has been induced by MMT. Even if there is a trend toward a higher sensitivity (i.e. lower withdrawal threshold in operated animals versus sham), this difference did not reach a statistical significance. Moreover, the narrow difference induced by OA does not allow placing a dose-response curve for an analgesic compound such as CR4056. Data are presented as mean \pm SEM.

Abbreviations: MMT, medial meniscal tear; OA, osteoarthritis; SD, Sprague Dawley.

Table SI Statistical analysis of HPWD in the MMT model of OA

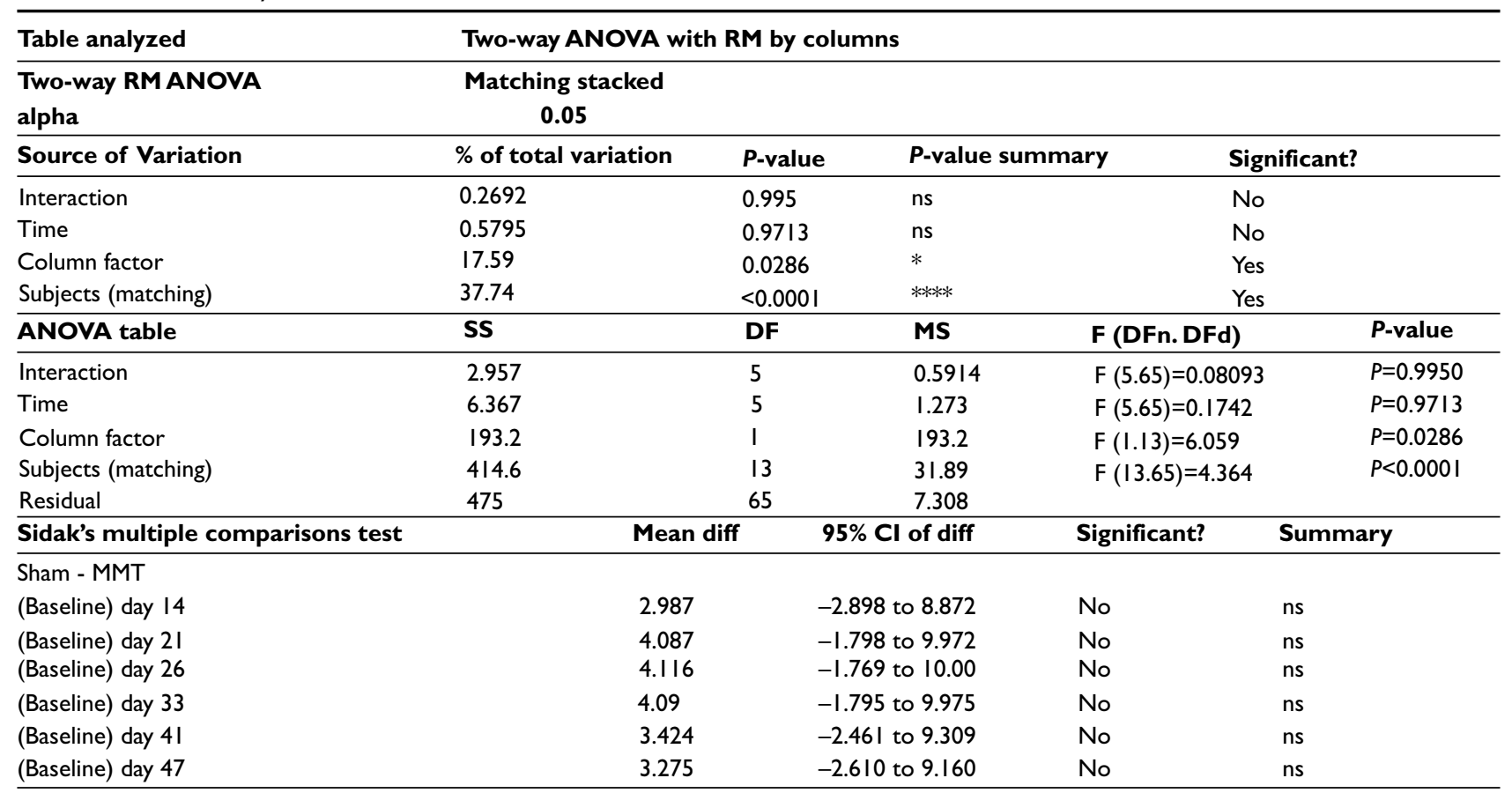

Notes: $* P<0.05 ; * * * * P<0.0001$.

Abbreviations: MMT, medial meniscal tear; OA, osteoarthritis; ANOVA, one-way analysis of variance, SEM, standard error of the mean; SD, Sprague Dawley; ns, not significant; RM, repeated measures; SS, sum of squares; DF, degrees of freedom; MS, mean square; $F$, variance of the group means; DFn, degrees of freedom numerator; DFd, degrees of freedom denominator. 


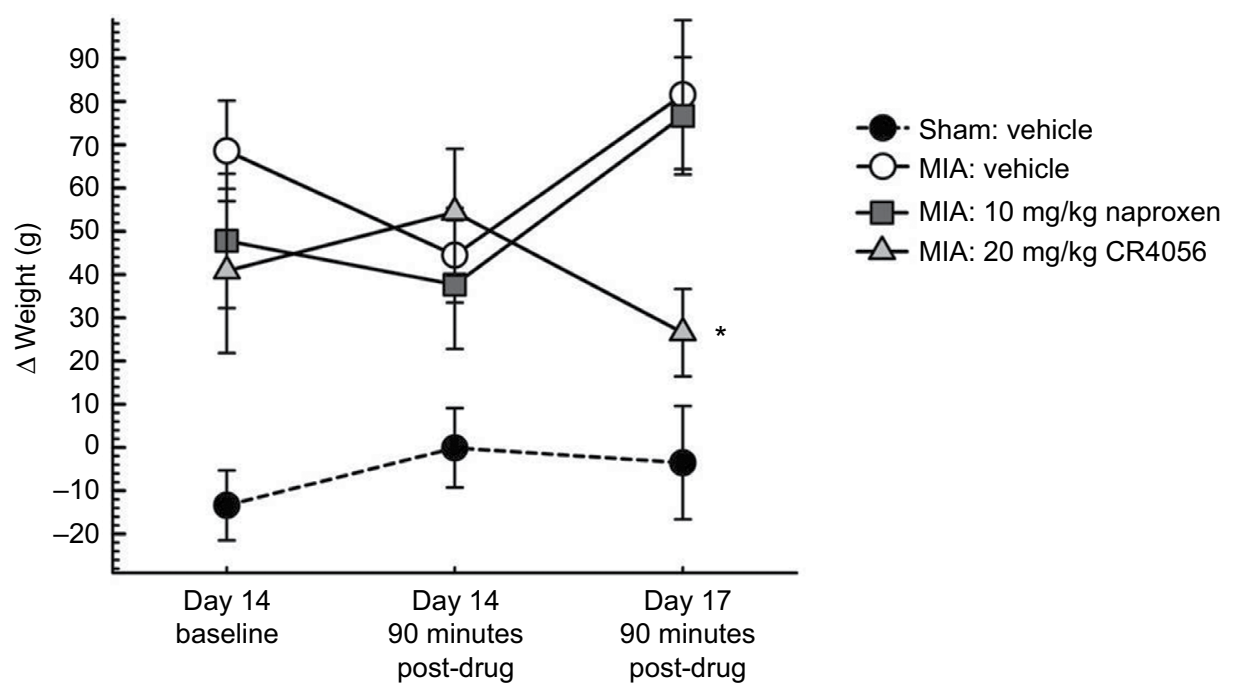

Figure S2 Determination of HPWD in the MIA model of OA in Wistar rats: effect of $20 \mathrm{mg} / \mathrm{kg}$ CR4056. HPWD has been evaluated as described. It is possible to observe the higher variability of HPWD (i.e. basal groups) compared to the MMT model. Nevertheless, also in this paradigm, CR4056 administration (20 mg/kg; oral, QD) induced a statistically significant reduction versus vehicle-treated animals. On the contrary, no effect is observed with naproxen (I0 mg/kg; oral, QD). Data are presented as mean $\pm \mathrm{SEM} ; * P<0.05$

Abbreviations: HPWD, hind paw weight distribution; MIA, monoiodoacetate; OA, osteoarthritis; SEM; standard error of the mean.

Table S2 Statistical analysis of HPWD in the MIA model of OA

\begin{tabular}{|c|c|c|c|c|c|}
\hline \multirow{2}{*}{$\begin{array}{l}\text { Table analyzed } \\
\text { Two-way RM ANOVA } \\
\text { alpha }\end{array}$} & \multicolumn{5}{|c|}{ Two-way ANOVA with RM by columns } \\
\hline & \multicolumn{5}{|l|}{$\begin{array}{l}\text { Matching stacked } \\
0.05\end{array}$} \\
\hline Source of Variation & $\%$ of total variation & $P$-value & $P$-value summary & Significant? & \\
\hline Interaction & 5.298 & 0.2316 & ns & No & \\
\hline Time & $\mathrm{I} .4 \mathrm{I}$ & 0.3685 & ns & No & \\
\hline Column factor & 19.95 & 0.0762 & ns & No & \\
\hline Subjects (matching) & 48.74 & 0.0986 & ns & No & \\
\hline ANOVA table & SS & DF & MS & F (DFn. DFd) & $P$-value \\
\hline Interaction & 2858 & 2 & 1429 & $F(2.15)=1.615$ & $P=0.2316$ \\
\hline Time & 760.8 & I & 760.8 & $F(I .15)=0.8598$ & $P=0.3685$ \\
\hline Column factor & 10,765 & 2 & 5382 & $F(2.15)=3.071$ & $P=0.0762$ \\
\hline Subjects (matching) & 26,293 & 15 & 1753 & $F(15.15)=1.981$ & $P=0.0986$ \\
\hline Residual & 13,273 & 15 & 884.9 & & \\
\hline Dunnett's multiple comparisons test & & Mean diff & $95 \% \mathrm{Cl}$ of diff & Significant? & Summary \\
\hline \multicolumn{6}{|l|}{ (Baseline) day 14} \\
\hline Vehicle versus naproxen (10 mg/kg) & & 20.77 & -27.90 to 69.43 & No & ns \\
\hline Vehicle versus CR4056 (20 mg/kg) & & 27.73 & -20.93 to 76.40 & No & ns \\
\hline \multicolumn{6}{|l|}{ Day $17+90$ minutes post-treatment } \\
\hline Vehicle versus naproxen ( 10 mg/kg) & & 4.9 & -43.76 to 53.56 & No & ns \\
\hline Vehicle versus CR4056 (20 mg/kg) & & 55.02 & -6.355 to 103.7 & Yes & $*$ \\
\hline
\end{tabular}

Notes: $* P<0.05$.

Abbreviations: HPWD, hind paw weight distribution; MIA, monoiodoacetate; OA, osteoarthritis; ANOVA, one-way analysis of variance, SEM, standard error of the mean; MMT, medial meniscal tear; ns, not significant; diff, difference; SS, sum of squares; DF, degrees of freedom; MS, mean square; F, variance of the group means; DFn, degrees of freedom numerator; DFd, degrees of freedom denominator.

\section{Journal of Pain Research}

\section{Publish your work in this journal}

The Journal of Pain Research is an international, peer reviewed, open access, online journal that welcomes laboratory and clinical findings in the fields of pain research and the prevention and management of pain. Original research, reviews, symposium reports, hypothesis formation and commentaries are all considered for publication.

\section{Dovepress}

The manuscript management system is completely online and includes a very quick and fair peer-review system, which is all easy to use. Visit http://www.dovepress.com/testimonials.php to read real quotes from published authors. 